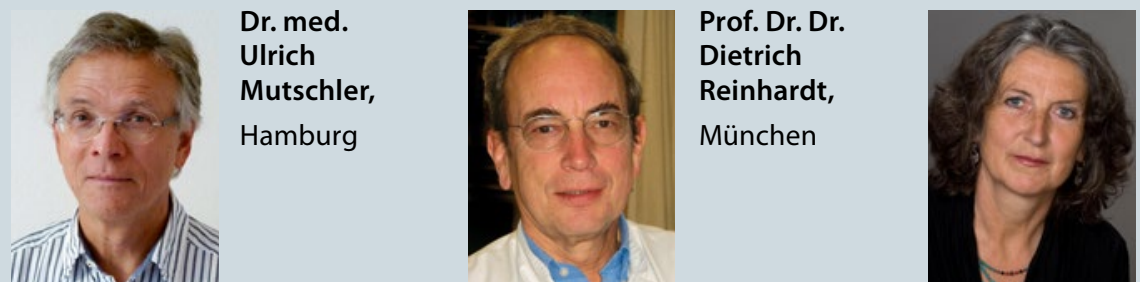

\section{Kommentar}

Nahrungsmittelallergien bei älteren Kindern und im Erwachsenenalter richten sich am häufigsten gegen pflanzliche Nahrungsmittel wie beispielsweise Nüsse, Leguminosen, Früchte und Gemüse. Eine Ausschaltung relevanter Allergene in der
Pflanze selbst stellt einen neuen Ansatz der Allergenkarenz zur Primär-, Sekundär- und Tertiärprävention von Nahrungsmittelallergien dar.

Zum aktuellen Zeitpunkt befindet sich die Entwicklung hypoallergener Nahrungsmittel mit gentechnischen Methoden aller-

\title{
Zusammenhang zwischen ADHS und Adipositas
}

\section{Schon lange wird vermutet, dass ADHS mit Übergewicht einhergeht. Eine aktuelle Metaanalyse konnte diese Beobachtungen nun bestätigen. Dabei wurde zusätzlich der Einfluss einer medikamentösen Therapie auf eine Adipositas bei ADHS-Patienten untersucht.}

$\mathrm{D}$ ie britischen Autoren einer aktuellen Studie gehen der Frage nach, ob es zwischen Aufmerksamkeitsdefizit-/Hyperaktivitätsstörung (ADHS) und Adipositas einen Zusammenhang gibt. Sie führten eine Metaanalyse mit 42 Studien durch: 728.136 Menschen, davon 48.161 mit ADHS, wurden erfasst. Sowohl bei Kindern (Odds Ratio [OR]: 1,20) als auch bei Erwachsenen (OR: 1,55) mit ADHS trat eine Adipositas signifikant häufiger auf als bei Probanden, die nicht unter ADHS litten. Die Prävalenz für Adipositas betrug bei Erwachsenen mit ADHS $28,2 \%$ versus $16,4 \%$ bei Gesunden, bei Kindern mit ADHS $10,4 \%$ versus $7,4 \%$. Wurde eine medikamentöse Therapie der ADHS durchgeführt, normalisierte sich das erhöhte Risiko für eine Adipositas.

Cortese $S$ et al. Association between ADHD and obesity: A systematic review and meta-analysis. Am J Psychiatry 2016;173(1):34-43

\section{Kommentar}

Die Metaanalyse stellt einen eindeutigen Zusammenhang zwischen ADHS und Adipositas her und zwar schon bei Kindern. Der Effekt bleibt auch noch im Erwachsenenalter erhalten. Offen bleibt die Kausalität: Diskutiert wird das Kernsymptom Impulsivität als Ursache. Möglich wäre auch eine primäre Störung im Belohnungszentrum, ein fehlendes "Sattheitsgefühl" oder die Folge der Außenseiterposition in Form von Frustessen. Wir beobachten auch immer wieder, dass erst in der Pubertät ein oft initial bestehendes Untergewicht in Adipositas umschlägt. Parallel dazu nimmt die Hyperaktivität ab und der visuelle Medienkonsum zu.

Eine Stimulanzientherapie verhindert die Entwicklung einer Adipositas. Die meist unerwünschte Nebenwirkung des Appetitmangels wirkt sich also protektiv aus. $\mathrm{Ob}$ dies allerdings auch bei Absetzen der Therapie anhält, geht aus den Studiendaten nicht hervor und erscheint aus der klini-
Dr. med. Kirsten Stollhoff, Hamburg dings noch in überwiegend präklinischen experimentellen Stadien. Bislang konnten schon erfolgreich Strategien zur stabilen Ausschaltung oder Reduktion der Expression von Allergenen in pflanzlichen Nahrungsmitteln etabliert werden.

Dr. Ulrich Mutschler

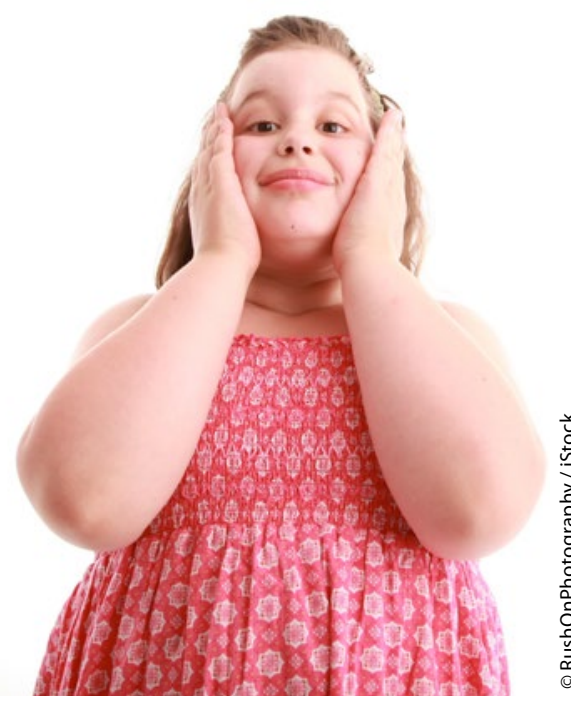

Bei Kindern mit ADHS besteht ein Risiko für Übergewicht.

schen Erfahrung als fraglich. Wenn die medikamentöse Therapie nicht durch extensiven Nikotinabusus ersetzt wird, erleben wir häufig eine deutliche Gewichtszunahme bei den Jugendlichen, die auf eigenen Wunsch die Medikation absetzen. Zur besseren Beurteilung der Kausalität und auch des Einflusses der medikamentösen Therapie sind Langzeitverläufe erforderlich. Auch fehlen Daten über den Gewichtsverlauf bei adipösen ADHS-Patienten unter medikamentöser Therapie. Dr. Kirsten Stollhoff 